\title{
Ellagic Acid Alleviates Hepatic Ischemia-Reperfusion Injury in C57 Mice via the Caspase-1-GSDMD Pathway
}

\section{Hao Wang}

Guizhou University

\section{Fujun Miao}

Yunnan Academy of Forestry and Grassland

Delu Ning

Yunnan Academy of Forestry and Grassland

Chunlan Shan ( $13614470751 @ 163 . c o m$ )

Guizhou University

\section{Research Article}

Keywords: Ellagic acid, Hepatic IRI, Pyroptosis, Caspase-1-GSDMD pathway

Posted Date: February 23rd, 2022

DOI: https://doi.org/10.21203/rs.3.rs-1286055/v1

License: (c) (i) This work is licensed under a Creative Commons Attribution 4.0 International License. Read Full License 


\section{Abstract \\ Background}

Ellagic acid (EA) is a phenolic component of some fruits and nuts, EA has protective properties against oxidative damage and inflammatory reaction in many disorders. Hepatic ischemia-reperfusion injury (IRI) is a common pathophysiological phenomenon in the veterinary clinic.

\section{Results}

In the present study, the protective effects of EA pretreatment against hepatic IRI-induced injury and the underlying mechanisms were investigated. Here, we found that pyroptosis is involved in hepatic IRI, which is manifested in increasing the expression of pyroptosis-related genes and promoting the expression of active caspase-1, thereby cleaving GSDMD-N to cause pyroptosis, and use caspase- $1^{-/-}$mice to verify this conclusion. In addition, we found that EA protects against hepatic IRI by inhibiting pyroptosis, including reducing the activity of caspase- 1 and its expression in the liver, inhibiting the lysis of GSDMD-N, and reducing the levels of IL-18 and IL-1 $\beta$.

\section{Conclusions}

The present results have demonstrated that prophylactic administration of EA ameliorated hepatic IRI by inhibiting pyroptosis induced in hepatic ischemia-reperfusion in vivo through the caspase-1-GSDMD axis, providing a potential therapeutic option prevent hepatic IRI in pets.

\section{Background}

In veterinary surgery, it is often necessary to operate on the liver. At this time, it is necessary to interrupt the blood supply of the liver. Restoration of the blood supply following ischaemia may lead to further liver damage, which is called hepatic ischemia-reperfusion injury (IRI) [1]. Blood flow restoration triggers tissue inflammation and ischemic damage by activating multi-protein complexes called inflammasomes [2]. Inflammasomes are related to the pathogenesis of hepatic IRI and are considered to be a key factor in liver cell damage [3]. After the inflammasome activation, the affected tissues undergo apoptosis and pyroptosis, another type of inflammation-related cell death $[4,5]$. Pyroptosis is a form of lytic programmed cell death initiated by GSDMD-N [6-8]. Although there is no direct evidence for the presence of pyroptosis in hepatic IRI, the activation of inflammasomes in hepatic IRI has been elucidated, indicating that pyroptosis is involved in hepatic IRI [9]. Interestingly, previous studies have shown that inhibiting pyroptosis can improve hepatic IRI and inhibit inflammation [10]. Before or during liver surgery, using drugs or small molecules to activate key survival pathways or inhibit pyroptosis pathways can help reduce hepatic IRI damage [11].

Ellagic acid is the dilactone of hexahydroxydibenzoic acid, and the name comes from the French word acide ellagique (Fig. 2A). EA is a naturally occurring polyphenolic compound that is found in many fruits, 
walnuts and plant extracts in the forms of hydrolysable tannins called ellagitannins, such as strawberries, grapes, pomegranates and walnuts [12-15]. As reported in previous studies, EA possesses antibacterial, anti-inflammatory, pneumoprotective, nephroprotective, and cardioprotective propertie [16].

Recent studies have shown that EA exerts a protective effect on the liver[17]. The research from Elyamany $M$. shows that EA treatment can significantly reduce the liver damage caused by valproic acid in rats [18], and L. Gu et al. demonstrated that EA protected against LPS/GalN-induced liver injury in SD rats by enhancing the antioxidative defense system and reducing the inflammatory response [19]. Kim, D described a significant reduction in endotoxemia and inflammatory liver damage with EA treatment by inhibiting the imbalance of the intestinal flora in c57 mice, elevated oxidative stress and apoptosis marker proteins [20]. However, the effect of EA on hepatic IRI remains unclear. In this study, the role of pyroptosis in hepatic ischemia-reperfusion injury was evaluated, illustrating that EA inhibits pyroptosis through the caspase-1GSDMD pathway, thereby protecting the liver from ischemia-reperfusion injury.

\section{Results}

\section{Caspase-1-GSDMD-induced pyroptosis occurs in the mice hepatic IRI model}

As shown in Fig. 2B, a mice hepatic IRI model was established. The effects of the operation on mice livers subjected to $90 \mathrm{~min}$ of warm ischemia followed by $2 \mathrm{~h}$ and $6 \mathrm{~h}$ of reperfusion were analyzed. As illustrated by the serum ALT (Fig. 2C), AST (Fig. 2D) and LDH (Fig. 2E) levels, reperfusion for 6 hours after ischemia caused more severe liver damage. The qPCR results showed that members of the CXC subfamily of chemokines, including CXCL-1, CXCL-2 and CXCL-10, were up-regulated in liver ischemia-reperfusion injury (Fig. 2F). As shown by histopathology (Fig. 2G), liver sections from mice undergoing hepatic IRI presented with significant features of severe centrilobular ballooning, congestion and lobular necrosis compared to those from sham-operated mice.

The pyroptosis induced by caspase-1-GSDMD could play a role in hepatic IRI. As shown in Fig. 3A to 3E, the expression of all inflammasome-associated genes in the liver tissues of the hepatic IRI mice model was assessed, with levels of NLRP3, ASC, Caspase-1, IL-1 $\beta$ and IL-18 transcripts being remarkably elevated in the hepatic IRI model mice, as compared with the sham group. Furthermore, the expression of caspase-1 increased in the liver of hepatic IRI model mice (Fig. 3F). Simultaneously, the serum levels of caspase-1 activity were up-regulated in hepatic IRI model mice (Fig. 3G). In addition, due to the cleavage of activated caspase- 1 and the formation of the activated N-terminal domain of GSDMD (GSDMD-N), it has recently been identified as the executioner of pyroptosis [21, 22]. We next examined whether hepatic IRI could induce proteolytic cleavage of GSDMD. The results confirmed that significant degrees of both caspase- 1 and GSDMD cleavage occurred in hepatic IRI (Fig. 3H). Conjointly, these data support the notion that Caspase-1GSDMD-induced pyroptosis occurs in the mice hepatic IRI model.

\section{Depletion of Caspase- 1 can reduce liver damage and inflammation caused by hepatic IRI}


Based on the findings that caspase-1-GSDMD mediated pyroptosis could play a role in hepatic IRI, caspase1-null background mice were used to further evaluate the role of pyroptosis in the pathogenesis of hepatic IRI. Specifically, the hepatic IRI or sham operation was performed on WT or Caspase-1-deficient (Caspase$1^{-/-}$) mice. WT mice displayed higher ALT, AST, and LDH levels in the hepatic IRI model as compared with sham groups. In contrast, the ALT, AST, and LDH levels of hepatic IRI Caspase-1 $1^{-/-}$mice were similar to those of the sham groups at $6 \mathrm{~h}$ of reperfusion (Fig. 4A, B, C). The histopathological examination further indicated that Caspase- $1^{-/-}$mice were spared from ischemia reperfusion-induced hepatic damage, as opposed to the hepatic IRI WT mice (Fig. 4D). Moreover, when the expression levels of proinflammatory cytokines IL-1 $\beta$ and IL-18 were measured in liver tissues, the depletion of caspase-1 abolished the increase of IL-1 $\beta$ and IL-18 of hepatic IRI model mice, as compared with their WT counterparts (Fig. 4E and F). In addition, our results also showed that the hepatic IRI model triggered cleavage of GSDMD, while caspase-1 ablation attenuated GSDMD cleavage, as assessed by immunoblotting analysis. This indicates the inhibition of pyroptosis in the mice livers (Fig. 4G). Accordingly, the liver damage and inflammation caused by hepatic IRI could be restrained by limiting Caspase-1-mediated pyroptosis.

\section{Ellagic acid reduce liver injury in hepatic ischemia- reperfusion mice by Inhibit pyroptosis}

The essential role of Caspase-1-GSDMD-mediated pyroptosis in hepatic IRI prompted the investigation of whether the Caspase-1-GSDMD pathway can be a potential therapeutic target for hepatic IRI. Studies have shown that EA has a protective effect on the liver. This study demonstrates that early treatment with EA can reduce the ALT, AST and LDH levels in the blood and reduce liver injury (Fig. 5A, B, C). Further histopathological examination revealed significant features of severe centrilobular ballooning, congestion and lobular necrosis in the hepatic IRI model mice group. However, these findings were potently attenuated after the EA treatment (Fig. 5D), suggesting that EA could reverse hepatic ischemia- reperfusion injury.

Furthermore, immunohistochemistry $(\mathrm{IHC})$ analysis of Caspase-1 specific markers for classic pyroptosis was carried out to assess hepatic pyroptosis. As shown in Fig. 5D, caspase-1 levels were significantly higher in the hepatic IRI model mice than in the sham group. Notably, EA administration robustly decreased the expression of Caspase- 1 to levels comparable to livers of sham mice. The serum levels of caspase-1 activity and mature IL-1 $\beta$ and IL-18 were up-regulated in the hepatic IRI model mice, whereas such an upregulation was repressed by EA treatment (Fig. 5E, F, G). Correspondingly, after EA treatment, the expression of pyroptosis-related genes, proteolytic cleavage of both caspase-1 and GSDMD was inhibited in the hepatic IRI model mice, indicating that EA indeed suppressed pyroptosis associated with hepatic IRI in vivo (Fig. S1, Fig. 5H). The above data suggest that Ellagic acid reduced liver injury in hepatic ischemiareperfusion mice by inhibiting pyroptosis.

\section{Discussion}

This study demonstrates that pyroptosis is involved in hepatic IRI, which is involved in increasing the expression of pyroptosis-related genes and promoting the expression of active Caspase-1, thereby cleaving GSDMD-N to cause pyroptosis. Importantly, we found that inhibition of pyroptosis by caspase- 1 ablation or 
EA treatment potently attenuates liver congestion and lobular necrosis caused by hepatic IRI. These findings reveal a previously unidentified underlying mechanism of hepatic ischemia reperfusion injury, providing a potential therapeutic option prevent hepatic IRI in pets.

Hepatic IRI is an inevitable complication in the pet's process of liver partial hepatectomy. It is an important cause of postoperative liver dysfunction and even liver failure. The mechanism of hepatic IRI has not been fully elucidated. In human medicine research, at present, it is considered that it may be the result of the joint action of many factors such as elevated free radicals, intracellular calcium overload, leukocyte activation, microvascular dysfunction and so on [23]. There are many forms of cell death involved in hepatic IRI, the main ones being apoptosis and necrosis. Autophagy, another form of programmed cell death, is also involved [24]. Hepatocyte damage and subsequent inflammation are the distinguishing features of hepatic IRI [10]. Pyroptosis is a form of programmed cell death that is different from apoptosis and necrosis and is dependent on inflammatory caspase-1 and/or caspase-11 in mice [25]. Although pyroptosis was first described in 1992 and later reported in 2001 [26, 27], the underlying mechanism of inflammasomemediated inflammatory caspase activation leading to lytic cell death remains unclear. GSDMD has been described as the executioner of pyroptosis [28-30]. In the absence of stimulation, full-length GSDMD remains intact, with the $\mathrm{N}$-terminal and $\mathrm{C}$-terminal regions interacting with each other. The oligomerized form GSDMD-N translocates to the plasma membrane and exhibits membrane-disrupting cytotoxicity in mammalian cells [31, 32]. Pyroptosis has been implicated in various diseases, such as nonalcoholic fatty liver, gastric cancer, and myocardial infarction $[33,34]$. However, whether pyroptosis plays a role in hepatic IRI remains unknown. This study demonstrates that the expression of pyroptosis-related genes is upregulated, and the activity of Caspase- 1 is increased, with an apparent activation of caspase-1-GSDMD in mice liver tissues during hepatic IRI, suggesting the possible involvement of pyroptosis in hepatic IRI. Moreover, depletion of caspase- 1 can reduce liver damage and inflammation caused by hepatic IRI. Pyroptosis can be induced by a canonical signaling pathway and a non-canonical signaling pathway [22, 35]. In this study, we found that the protein levels and the activity of caspase- 1 were remarkably enhanced during hepatic IRI, suggesting the activation of the canonical pyroptosis signaling pathway (caspase-1GSDMD pathway).

EA is a naturally occurring polyphenolic compound that is found in many fruits and walnuts [16, 36]. Studies have shown that EA protects endothelial cells from apoptosis induced by oxidized low-density lipoprotein by regulating the $\mathrm{PI} 3 \mathrm{~K} / \mathrm{Akt} / \mathrm{eNOS}$ pathway[37], and also has antibacterial and anti-inflammatory effects [38]. Furthermore, EA can reduce the pulmonary toxicity induced by bleomycin and cyclophosphamide in Wistar rats [39], and has a renal protective effect on cisplatin nephrotoxic rats [40]. Recent studies have shown that EA has a protective effect on the liver [18], as confirmed by our results. Caspase-1-GSDMD-mediated pyroptosis plays an important role in hepatic IRI, but the protective molecular mechanism of EA in hepatic ischaemic-reperfusion injury needs further investigation. Serum ALT and AST are widely used as markers of liver injury [19]. In this study, EA (60 mg/kg) was administered to mice for 14 consecutive days before surgery. The serum ALT, AST and LDH levels were measured, as well as liver histopathology to study the effects of ellagic acid on ischemia-reperfusion. The results show that ellagic acid can significantly reduce the liver injury caused by ischemia-reperfusion, which is manifested in the 
decrease of ALT and AST levels. In addition, histological analysis showed that ellagic acid could significantly reduce liver damage. Because EA can protect hepatic IRI, and Caspase-1-GSDMD-mediated pyroptosis is involved in hepatic IRI, we are interested in whether EA can protect hepatic IRI by inhibiting pyroptosis. The results show that fewer caspase- 1 expressions were detected in the livers of EA-intervened mice. The caspase- 1 activity and mature IL-1 $\beta$ and IL-18 levels were up-regulated in hepatic IRI model mice, whereas the same was repressed by EA treatment. Correspondingly, after EA treatment, proteolytic cleavage of both caspase-1 and GSDMD was inhibited in the hepatic IRI model mice, indicating that EA indeed suppressed pyroptosis associated with hepatic IRI in vivo. These evidence indicate that ellagic acid can protect against hepatic IRI by inhibiting the molecular mechanism of the caspase-1-GSDMD pathway.

In summary, we found that Ellagic acid ameliorated hepatic IRI-induced injury by inhibiting pyroptosis in vivo through the caspase-1-GSDMD pathway(The schematic diagram is shown in Fig. 6).

\section{Conclusions}

This is the first study to inhibit Caspase-1-GSDMD-induced pyroptosis through dietary supplementation of ellagic acid, thereby protecting the liver from ischemia-reperfusion injury. The results demonstrated that the pyroptosis triggered by Caspase-1-GSDMD is involved in liver ischemia-reperfusion injury. In this study, prophylactic supplementation of EA for 2 weeks inhibited pyroptosis and improved liver function during hepatic ischemia-reperfusion injury. These new findings provide basic information that EA may regulate cell pyroptosis in liver ischemia-reperfusion injury, which may help reduce liver inflammation and injury in pet with ischemia-reperfusion. The results of this study provide basic information and suggest that prophylactic use of EA may be protective for pet with liver ischemia-reperfusion injury (such as pet after process of liver partial hepatectomy).

\section{Materials And Methods}

\section{Animals}

Male wild-type and Caspase-1 ${ }^{-/-}$C57BL/ 6 mice(18-22g) were purchased from Cyagen (Wuhan, China). The mice were kept under specific pathogen-free conditions, and the cages were located in a room with an alternating photoperiod of $12 \mathrm{~h}$ light and $12 \mathrm{~h}$ darkness, a temperature of $25^{\circ} \mathrm{C} \pm 2^{\circ} \mathrm{C}$, and ambient humidity $(40-60 \%)$. The mice were then randomly assigned to experimental animal groups, and the experimental protocol was approved by the Animal Ethics Committee of Guizhou University.

\section{Hepatic IRI mice model and treatment}

As described in previous studies [41], a warm partial hepatic IRI model was used. In short, the control group (sham intervention, $n \geq 5$ ) underwent laparotomy, but the hepatic portal veins were not obstructed. The mice in the hepatic IRI group were freed from the hepatic portal vein and blocked the blood supply to the mid lobe and left-hepatic lobe for an hour and a half, and the blood vessels were then opened for $2 \mathrm{~h}$ or $6 \mathrm{~h}$. If the mice died before sampling, the sample was discarded. All operations were performed by the same operator, and the mice were allowed to fast for 14 hours before the operation. Mice in the treatment group were subjected 
to oral administration of a daily dose of $60 \mathrm{mg} / \mathrm{kg}$ EA (Solarbio, Beijing, China) for 14 days before performing surgery to model liver ischemia [20]. Experiment design is illustrated in Fig. 1.

\section{Biochemical measurement}

Blood was collected from the mice eyeballs and centrifuged at 8,000 rpm for 10 mins. Serum ALT, AST, and LDH levels were measured by microplate test kits (Jiancheng Bioengineering Institute, Nanjing, China).

\section{Histopathology and Immunohistochemistry}

Liver tissues were fixed with $4 \%$ paraformaldehyde overnight at $4^{\circ} \mathrm{C}$, followed by dehydration through an alcohol-xylene series, and finally embedded in paraffin. Livers ections ( $5 \mu \mathrm{m}$ thickness) were cut (Leica2235, GER), dried at $37^{\circ} \mathrm{C}$, deparaffinized, rehydrated through a series of xylene-alcohol, rinsed with deionized water, and finally stained with H\&E(Hematoxylin and Eosin).

With reference to UltraSensitive ${ }^{\mathrm{TM}}$ SP (Mice/Rabbit) IHC Kit (MXB, China), the slices were prepared in $0.01 \mathrm{M}$ citrate buffer ( $\mathrm{pH}$ 6.0) for antigen retrieval. The slices were immersed in $3 \% \mathrm{H}_{2} \mathrm{O}_{2}$ for 10 minutes, and the non-immune goat serum was blocked for 10 minutes at room temperature. Subsequently, the solution was incubated with caspase-1 (Santa Cruz, 14F468) at $4^{\circ} \mathrm{C}$ for about 12 hours. The slices were washed 3 times with PBS (MXB, China) buffer and then placed with the secondary antibody at room temperature for 30 minutes. Subsequently, the sections were stained with DAB ( $M X B$, China) for 5 minutes and then with hematoxylin for 10 minutes. The images were captured using the Olympus BX43F microscope (Tokyo, Japan). The caspase-1 positive areas were measured using ImageJ.

\section{Western blotting analysis}

The western blotting experiment was carried out according to protocol [42]. Primary antibodies were used against mice caspase-1 (\#ab179515) (Abcam, MA, USA), GSDMD (\#ab209845) (Abcam, MA, USA). For these specific proteins, $\beta$-actin (Sigma-Aldrich St. Louis, MO, USA) was used as a loading control.

\section{RNA extraction and real-time PCR}

The total RNA was extracted from mice brain tissues with an RNAiso Plus kit (Takara, Dalian, China) according to the manufacturer's instructions. One microgram of total RNA was reverse-transcribed to cDNA using the PrimeScript RT Master Mix kit (Takara, Dalian, China). Real-time PCR reactions were performed in triplicate using SYBR Premix Ex Taq II (Takara, Dalian, China) and analyzed with CFX96TM Real-time System (Bio-Rad, Hercules, CA, USA). The relative changes in mRNA were calculated using the $\Delta \Delta \mathrm{Ct}$ method and standardized to housekeeping gene $\beta$-actin. The sequences of the primers used are provided in Table 1. 
Table 1

Primers used for GPCR analysis.

\begin{tabular}{|c|c|c|c|}
\hline Name & Sequences $\left(5^{\prime}-3^{\prime}\right)$ & Name & Sequences $\left(5^{\prime}-3^{\prime}\right)$ \\
\hline$\beta$-actin & CCTGCGGCATTCACGAAACTAC & \multirow{2}{*}{$\begin{array}{l}\text { IL-18 } \\
\text { BC024384.1 }\end{array}$} & GGCTGCCATGTCAGAAGACT \\
\hline XM_005887322.2 & АСTCCTGCTTGCTGATCCACAAT & & CCTCGAACACAGGCTGTCTT \\
\hline NLRP3 & AACTGCAGCATCTCCTGGAC & \multirow{2}{*}{$\begin{array}{l}\text { CXCL-1 } \\
\text { NM_008176.3 }\end{array}$} & TTCACСTCAAGAACATCCAG \\
\hline KF032621.1 & ACACAATCCAGCAGACCAGG & & TACTTGGGGACACCTTTTAG \\
\hline ASC & AACCCAAGCAAGATGCGGAAG & \multirow{2}{*}{$\begin{array}{l}\text { CXCL-2 } \\
\text { NM_009140.2 }\end{array}$} & CTTCAAGAACATCCAGAGCT \\
\hline AB059327.1 & TTAGGGCCTGGAGGAGCAAG & & ATGATTTTCTGAACCAGGGG \\
\hline Caspase-1 & GCCGTGGAGAGAAACAAGGA & \multirow{2}{*}{$\begin{array}{l}\text { CXCL-10 } \\
\text { NM_021274.2 }\end{array}$} & ACTGCATCCATATCGATGAC \\
\hline BC008152.1 & AAAAGTGAGCCCCTGACAGG & & СTTTTTCATCGTGGCAATGA \\
\hline IL-1 $\beta$ & AATGAAAGACGGCACACCCA & & \\
\hline NM_008361.4 & GGAAGACAGGCTTGTGCTCT & & \\
\hline
\end{tabular}

\section{ELISA assay}

ELISA kits were used to detect mice serum levels of IL-18 and IL-1 (Jiancheng Bioengineering, Nanjing, China) according to the manufacturer's protocols.

\section{Caspase-1 activity}

Caspase-1 activity was detected in liver tissues. The activity was measured with caspase-1 Assay Kit (Jiancheng Biotechnology, Nanjing, China) according to the manufacturer's instructions.

\section{Statistical analysis}

GraphPad Prism 8.0 software (GraphPad Software, San Diego, CA, USA) was used for statistical analysis. The results are expressed as mean \pm SEM unless otherwise stated. The unpaired Student's t-test was used to compare the two groups, and the variances between the statistically compared groups were similar. A $P$ value of $<0.05$ was considered significant.

\section{Declarations}

\section{Ethics approval and consent to participate}

The authors confirm that the ethical policies of the journal, as noted on the journal's author guidelines page, have been adhered to and the appropriate ethical review committee approval has been received. All experiments were approved by the Animal Ethics Committee of Guizhou University (EAE-GZU-2021-T096) committee.The study was carried out in compliance with the ARRIVE guidelines. 
Not applicable

\section{Availability of data and materials}

The datasets generated during and/or analyzed during the current study are available from the corresponding author on reasonable request.

ß-actin(XM_005887322.2, https://www.ncbi.nlm.nih.gov/search/all/?term=XM_005887322.2 ),

NLRP3(KF032621.1, https://www.ncbi.nlm.nih.gov/search/all/?term=KF032621.1),

ASC(AB059327.1, https://www.ncbi.nlm.nih.gov/search/all/?term=AB059327.1),

Caspase-1(BC008152.1, https://www.ncbi.nlm.nih.gov/search/all/?term=BC008152.1),

IL-1ß(NM_008361.4, https://www.ncbi.nlm.nih.gov/search/all/?term=NM_008361.4),

IL-18(BC024384.1, https://www.ncbi.nlm.nih.gov/search/all/?term=BC024384.1),

CXCL-1(NM_008176.3, https://www.ncbi.nlm.nih.gov/search/all/?term=NM_008176.3),

CXCL-2(NM_009140.2, https://www.ncbi.nlm.nih.gov/search/all/?term=NM_009140.20),

CXCL-10(NM_021274.2, https://www.ncbi.nlm.nih.gov/search/all/?term=NM_021274.2).

All data can be accessed in the National Center for Biotechnology Information.

\section{Competing interests}

The authors declare that they have no competing interests.

\section{Funding}

This research work was financially supported by the Cultivation project of Guizhou University (Grant No. 202011); The Science and Technology Major Program of Yunnan Province, China (Grant No. 2018ZG003).

\section{Acknowledgments}

This research work was financially supported by the Cultivation project of Guizhou University (Grant No. 202011); The Science and Technology Major Program of Yunnan Province, China (Grant No. 2018ZG003).

\section{Author Contributions}

Hao Wang: Conceptualization, Methodology, Investigation, Formal analysis, Writing - original draft, Writing - review \& editing. Fujun Miao: Methodology, Investigation, Formal analysis, Writing - review \& editing. Delu Ning: Conceptualization, Supervision, Funding acquisition. Chunlan Shan: Conceptualization, Supervision, Funding acquisition. All authors reviewed the manuscript. 


\section{References}

1. Saidi R, Kenari SJJoistojotAoSR: Liver ischemia/reperfusion injury: an overview. 2014, 27(6):366-379.

2. Mohamadi Y, Mousavi M, Khanbabaei H, Salarinia R, Javankiani S, Hassanzadeh G, Momeni FJJocb: The role of inflammasome complex in ischemia-reperfusion injury. 2018.

3. Szabo G, Petrasek JJNrG, hepatology: Inflammasome activation and function in liver disease. 2015, 12(7):387-400.

4. Wu C, Lu W, Zhang Y, Zhang G, Shi X, Hisada Y, Grover S, Zhang X, Li L, Xiang B et al: Inflammasome Activation Triggers Blood Clotting and Host Death through Pyroptosis. 2019, 50(6):1401-1411.e1404.

5. Christgen S, Place D, Kanneganti TJCr: Toward targeting inflammasomes: insights into their regulation and activation. 2020, 30(4):315-327.

6. Shi J, Gao W, Shao FJTibs: Pyroptosis: Gasdermin-Mediated Programmed Necrotic Cell Death. 2017, 42(4):245-254.

7. Chen X, He W, Hu L, Li J, Fang Y, Wang X, Xu X, Wang Z, Huang K, Han JJCr: Pyroptosis is driven by non-selective gasdermin-D pore and its morphology is different from MLKL channel-mediated necroptosis. 2016, 26(9):1007-1020.

8. Liu Z, Wang C, Yang J, Zhou B, Yang R, Ramachandran R, Abbott D, Xiao TJI: Crystal Structures of the Full-Length Murine and Human Gasdermin D Reveal Mechanisms of Autoinhibition, Lipid Binding, and Oligomerization. 2019, 51(1):43-49.e44.

9. Kim H, Kim S, Lee SJTFj: Activation of NLRP3 and AIM2 inflammasomes in Kupffer cells in hepatic ischemia/reperfusion. 2015, 282(2):259-270.

10. Li J, Zhao J, Xu M, Li M, Wang B, Qu X, Yu C, Hang H, Xia Q, Wu H et al: Blocking GSDMD processing in innate immune cells but not in hepatocytes protects hepatic ischemia-reperfusion injury. 2020, 11(4):244.

11. Yang W, Chen J, Meng Y, Chen Z, Yang JJljoms: Novel Targets for Treating Ischemia-Reperfusion Injury in the Liver. 2018, 19(5).

12. Soong YY, Ba Rlow PJJFC: Quantification of gallic acid and ellagic acid from longan (Dimocarpus longan Lour.) seed and mango (Mangifera indica L.) kernel and their effects on antioxidant activity. 2006, 97(3):524-530.

13. Plundrich N, Grace MH, Raskin I, Lila MAJIJoCS: Bioactive polyphenols from muscadine grape and blackcurrant stably concentrated onto protein-rich matrices for topical applications. 2013, 35(4):394401.

14. Sánchez-González C, Ciudad C, Noé V, Izquierdo-Pulido MJCrifs, nutrition: Health benefits of walnut polyphenols: An exploration beyond their lipid profile. 2017, 57(16):3373-3383.

15. Türk G, Sönmez M, Ceribaşi A, Yüce A, Ateşşahin AJli: Attenuation of cyclosporine A-induced testicular and spermatozoal damages associated with oxidative stress by ellagic acid. 2010, 10(2):177-182.

16. García-Niño W, Zazueta CJPr: Ellagic acid: Pharmacological activities and molecular mechanisms involved in liver protection. 2015, 97:84-103. 
17. Lee J, Won J, Choi J, Cha H, Jang Y, Park S, Kim H, Kim H, Kim DJJoa, chemistry f: Protective effect of ellagic acid on concanavalin A-induced hepatitis via toll-like receptor and mitogen-activated protein kinase/nuclear factor KB signaling pathways. 2014, 62(41):10110-10117.

18. Elyamany M, Abdelkader NF, Gad AM, Assaf N, Elesawy WHJJoPS: Ellagic acid attenuates liver toxicity induced by valproic acid in rats. 2020, 143(1).

19. Gu L, Deng WS, Liu Y, Jiang CH, Sun LC, Sun XF, Xu Q, Zhou HJIl: Ellagic acid protects Lipopolysaccharide/d-galactosamine-induced acute hepatic injury in mice. 2014, 22(2):341-345.

20. Kim D, Sim Y, Hwang J, Kwun I, Lim J, Kim J, Kim J, Baek M, Akbar M, Seo W et al: Ellagic Acid Prevents Binge Alcohol-Induced Leaky Gut and Liver Injury through Inhibiting Gut Dysbiosis and Oxidative Stress. 2021, 10(9).

21. He WT, Wan H, Hu L, Chen P, Wang X, Huang Z, Yang ZHJCR: Gasdermin D is an executor of pyroptosis and required for interleukin-1 $\beta$ secretion. 2015, 25(012):1285-1298.

22. Liu X, Zhang Z, Ruan J, Pan Y, Magupalli VG, Wu H, Lieberman JJN: Inflammasome-activated gasdermin $D$ causes pyroptosis by forming membrane pores. 2016, 535(7610):153.

23. Hirao H, Nakamura K, Kupiec-Weglinski JJNrG, hepatology: Liver ischaemia-reperfusion injury: a new understanding of the role of innate immunity. 2021.

24. Domart M, Esposti D, Sebagh M, Olaya N, Harper F, Pierron G, Franc B, Tanabe K, Debuire B, Azoulay D et al: Concurrent induction of necrosis, apoptosis, and autophagy in ischemic preconditioned human livers formerly treated by chemotherapy. 2009, 51(5):881-889.

25. Aachoui Y, Leaf IA, Hagar JA, Fontana MF, Campos CG, Zak DE, Tan MH, Cotter PA, Vance RE, Aderem AJS: Caspase-11 Protects Against Bacteria That Escape the Vacuole. 2013, 339(6122):975-978.

26. Cookson BT, Brennan MAJTiM: Pro-inflammatory programmed cell death. 2001, 9(3):113-114.

27. Jorgensen I, Miao EJIr: Pyroptotic cell death defends against intracellular pathogens. 2015, 265(1):130-142.

28. Li Z, Liu W, Fu J, Cheng S, Xu Y, Wang Z, Liu X, Shi X, Liu Y, Qi X et al: Shigella evades pyroptosis by arginine ADP-riboxanation of caspase-11. 2021.

29. Hou J, Hsu J, Hung MJMc: Molecular mechanisms and functions of pyroptosis in inflammation and antitumor immunity. 2021.

30. Shi J, Zhao Y, Wang K, Shi X, Wang Y, Huang H, Zhuang Y, Cai T, Wang F, Shao FJN: Cleavage of GSDMD by inflammatory caspases determines pyroptotic cell death. 2015, 526(7575):660-665.

31. Ding J, Wang K, Liu W, She Y, Sun Q, Shi J, Sun H, Wang DC, Shao FJN: Pore-forming activity and structural autoinhibition of the gasdermin family. 2016.

32. Zhang Y, Xin C, Gueydan C, Han JJCR: Plasma membrane changes during programmed cell deaths. 2018, 28(001):9-21.

33. Stefano T, Mauro AG, S CZ, Antonio AJAH, Physiology C: Inflammasome, pyroptosis, and cytokines in myocardial ischemia-reperfusion injury. 2018, 315:ajpheart.00158.02018-.

34. Xu B, Jiang M, Chu Y, Wang W, Chen D, Li X, Zhang Z, Zhang D, Fan D, Nie YJJoH: Gasdermin D plays a key role as a pyroptosis executor of non-alcoholic steatohepatitis in humans and mice. 
2017:S0168827817324947.

35. Miao EA, Rajan JV, Aderem AJIR: Caspase-1-induced pyroptotic cell death. 2011, 243(1):206-214.

36. Regueiro J, Sánchez-González C, Vallverdú-Queralt A, Simal-Gándara J, Lamuela-Raventós R, IzquierdoPulido MJFc: Comprehensive identification of walnut polyphenols by liquid chromatography coupled to linear ion trap-Orbitrap mass spectrometry. 2014, 152:340-348.

37. Ou H, Lee W, Lee S, Huang C, Chiu T, Tsai K, Hsu W, Sheu WJT, pharmacology a: Ellagic acid protects endothelial cells from oxidized low-density lipoprotein-induced apoptosis by modulating the PI3K/Akt/eNOS pathway. 2010, 248(2):134-143.

38. Abuelsaad A, Mohamed I, Allam G, Al-Solumani AAJLS: Antimicrobial and immunomodulating activities of hesperidin and ellagic acid against diarrheic Aeromonas hydrophila in a murine model. 2013, 93(20):714-722.

39. Saba, Khan S, Parvez S, Chaudhari B, Ahmad F, Anjum S, Raisuddin SJF, Toxicology C: Ellagic acid attenuates bleomycin and cyclophosphamide-induced pulmonary toxicity in Wistar rats. 2013, 58:210219.

40. El-Garhy AM, El-Raouf OA, El-Sayeh BM, Fawzy HM, Abdallah DMJJBMT: Ellagic Acid Antiinflammatory and Antiapoptotic Potential Mediate Renoprotection in Cisplatin Nephrotoxic Rats. 2015, 28(10):472479.

41. Zhu J, Lu T, Yue S, Shen X, Yuan ZJT: Rapamycin protection of livers from ischemia and reperfusion injury is dependent on both autophagy induction and mammalian target of rapamycin complex 2-Akt activation. 2014, 99(1).

42. He Z, An S, Chen J, Zhang S, Tan C, Yu J, Ye H, Wu Y, Yuan J, Wu J: Neural progenitor cell pyroptosis contributes to Zika virus-induced brain atrophy and represents a therapeutic target. 2020.

\section{Figures}


Male WT C57BL/6 mice or Caspase-1/- C57BL/6 mice

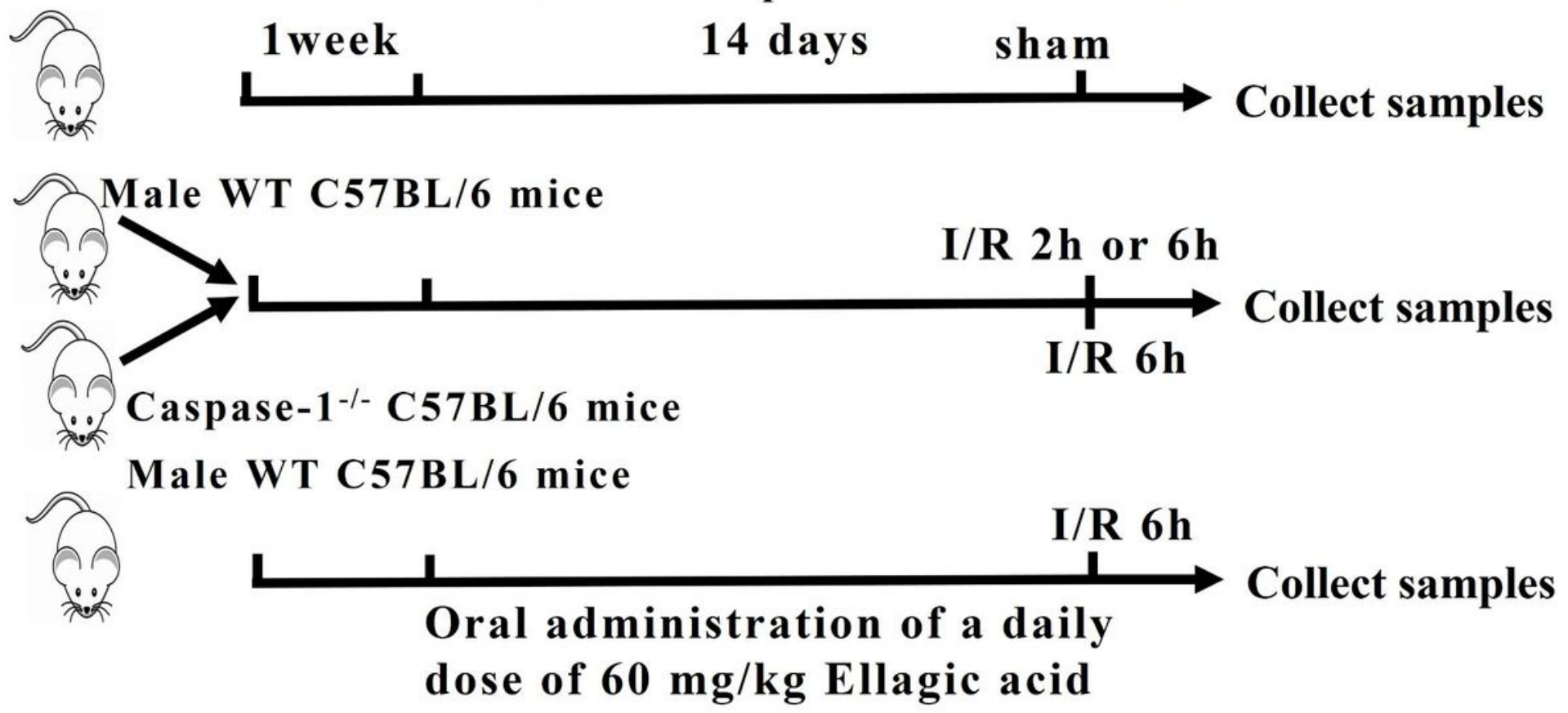

Figure 1

Summary of experiments design. 
A<smiles>O=c1oc2c(O)c(O)cc3c(=O)oc4c(O)c(O)cc1c4c23</smiles>

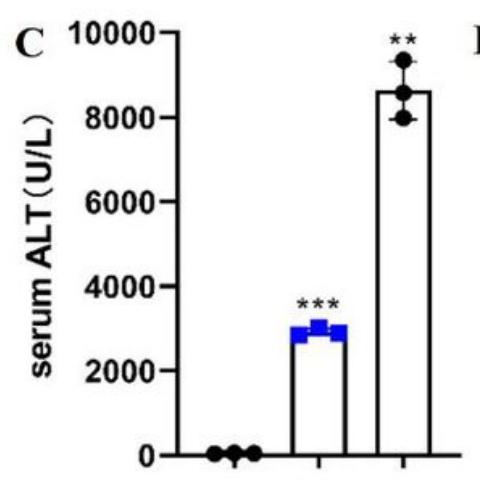

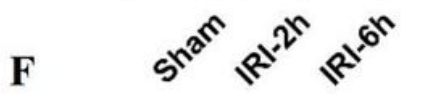

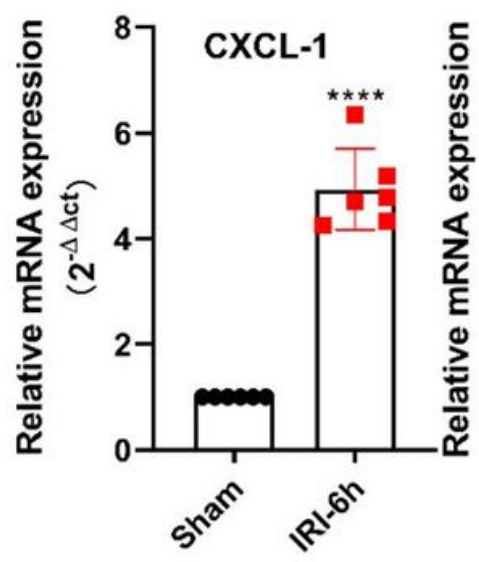

B
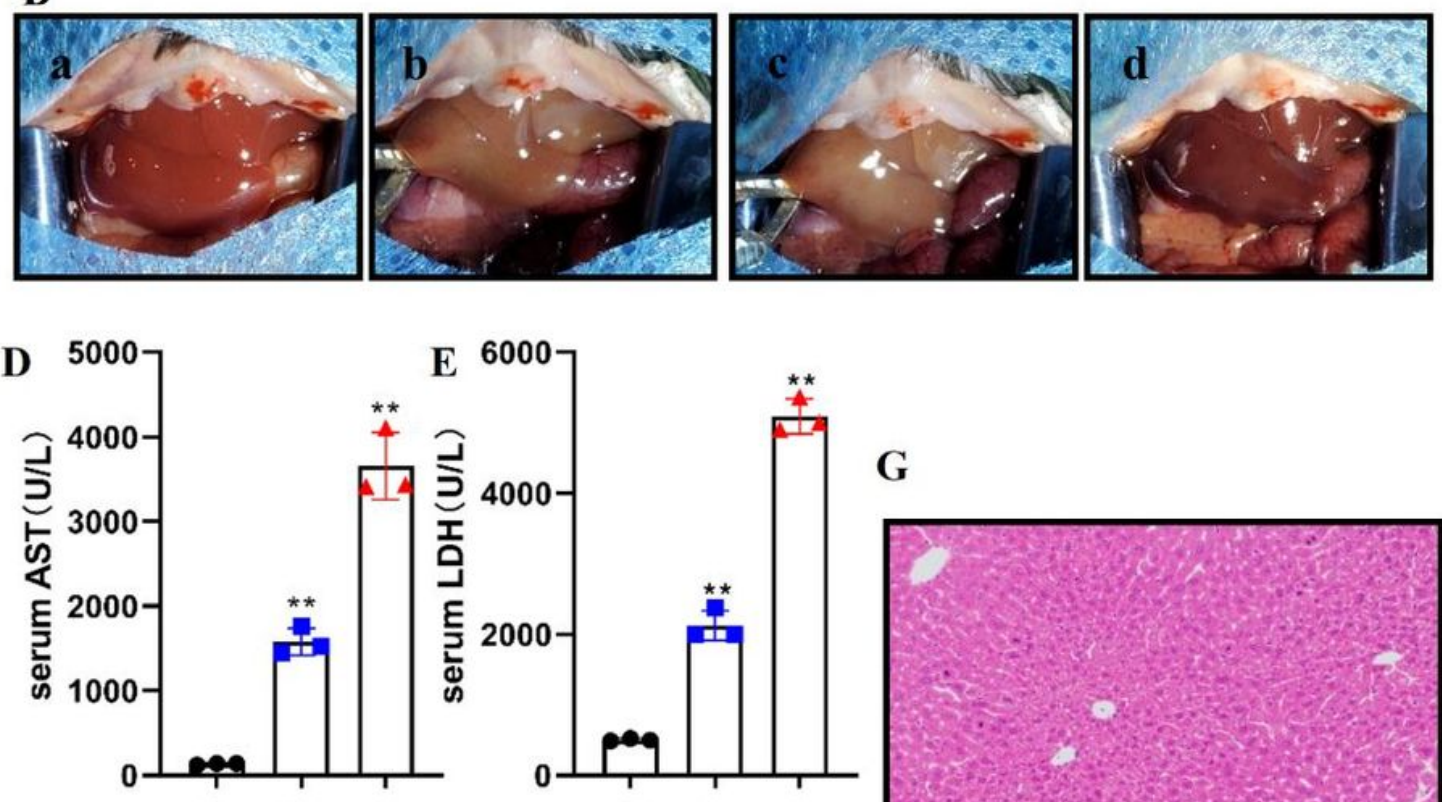

50

E ${ }^{6000}$

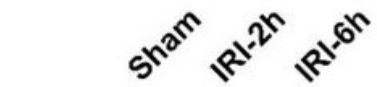
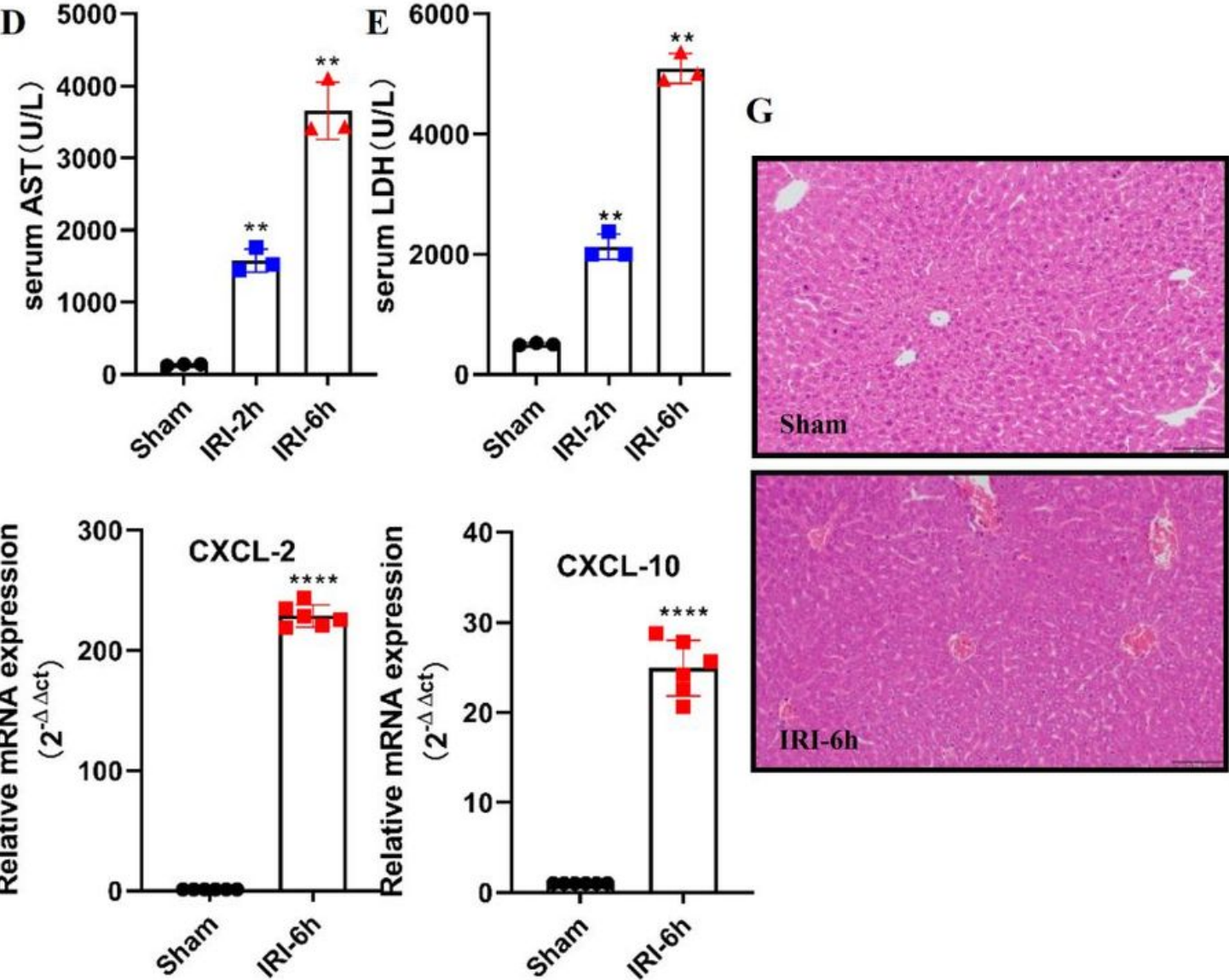

IRI-6h

\section{Figure 2}

Severe liver damage caused by ischemia-reperfusion. (A) Chemical structure of ellagic acid. (B) C57/B6 mice were subjected to hepatic IRI model (a. before hepatic ischemia, b. start hepatic ischemia, c. ischemia for $90 \mathrm{~min}$, d. reperfusion). (C), (D) and (E) The hepatocellular function in serum samples was evaluated by detecting ALT, AST, and LDH levels $(n=3)$. (F) The relative expression levels of CXCL-1, CXCL-2 and CXCL10 were determined using qRT-PCR in mice liver specimens $(n=6)$. (G) Representative images of hematoxylin and eosin (HE) staining (scale bar, $100 \mu \mathrm{m}$ ). All data are shown as the mean \pm SD. ${ }^{\star \star \star \star} P<$ $0.0001,{ }^{\star} P<0.01$. 

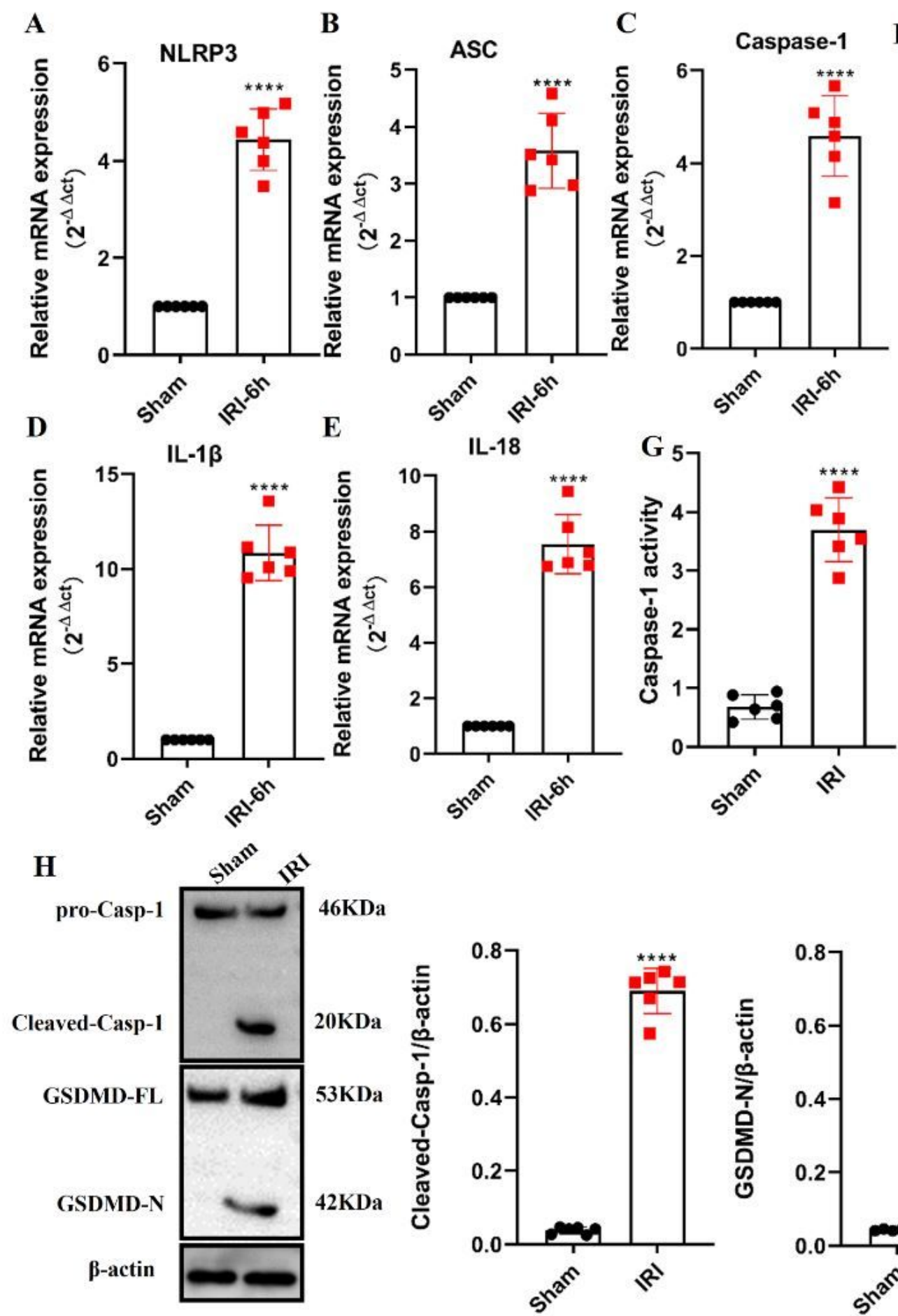

46KDa
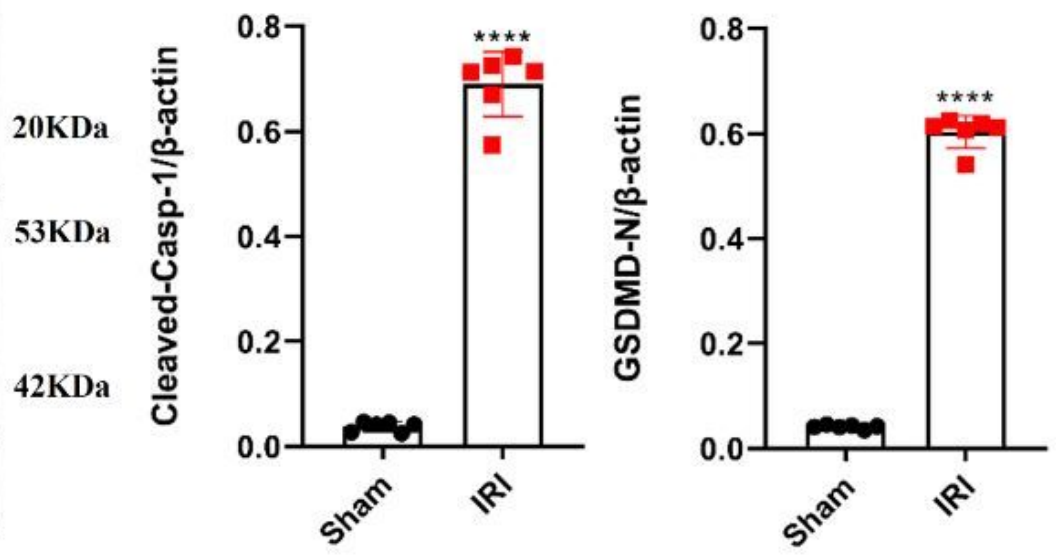

Figure 3

Caspase-1-GSDMD-induced pyroptosis occurs in the mice hepatic IRI model. (A-E) Relative expression levels of inflammasome-associated genes were determined using qRT-PCR in mice liver specimens $(n=6)$. (F) Representative images of caspase-1 staining (scale bar, $100 \mu \mathrm{m}$ ), the area of Caspase-1-positive cells in the livers were also determined $(n=6)$. (G) Caspase-1 activities were measured in mice hepatic IRI liver tissues $(n=6) .(H)$ Examination of the proteolytic cleavage of Caspase-1 and GSDMD in mice liver specimens with Hepatic IRI or sham intervention, using immunoblotting analysis. GSDMD-FL, full-length 
GSDMD; GSDMD-N, the N-terminal cleavage product of GSDMD $(n=6)$. All data are shown as the mean \pm SD. ${ }^{* \star \star \star} P<0.0001{ }^{\star \star * \star} P<0.001$.

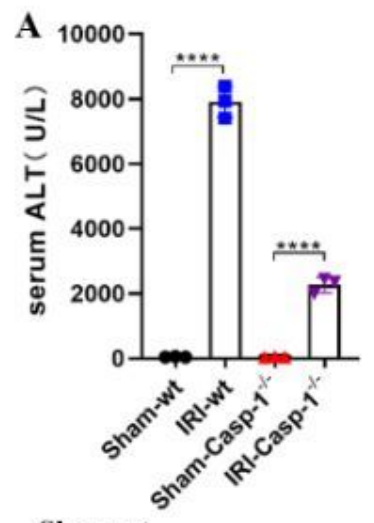

D

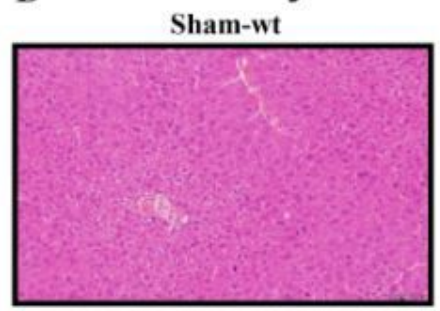

Sham-Casp-1-/-
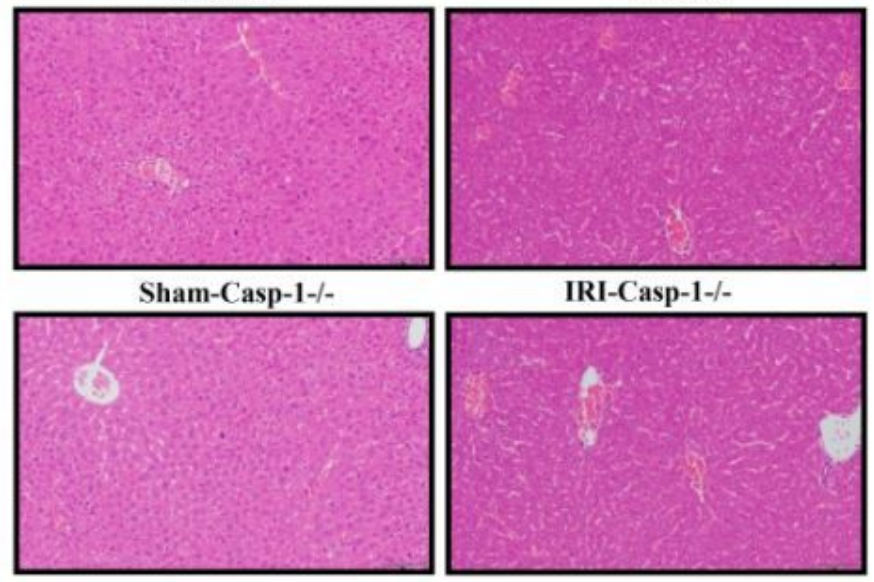

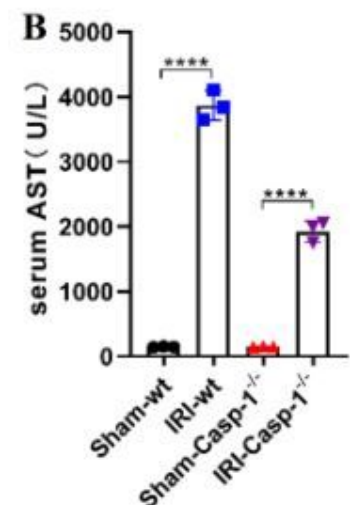

IRI-wt

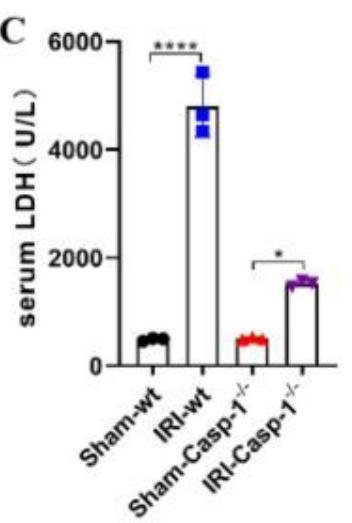

E

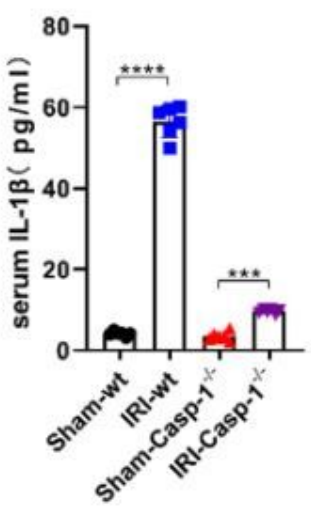

F
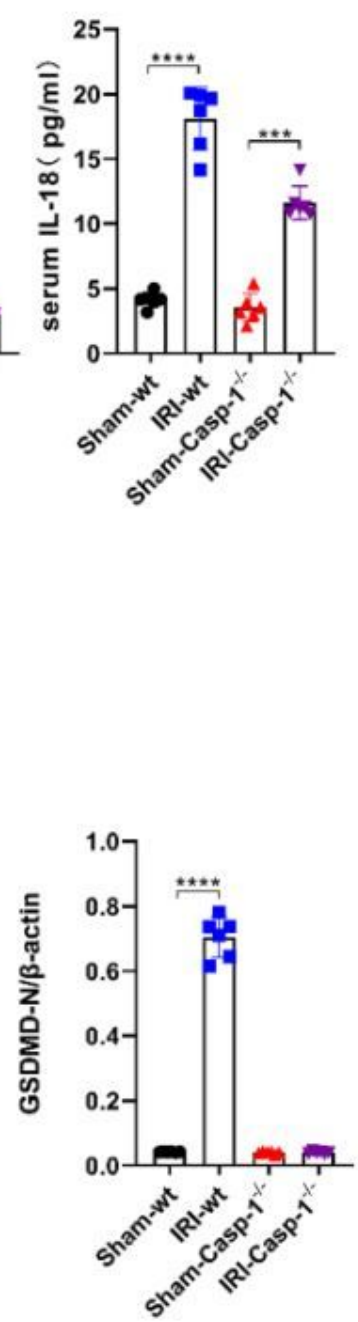

Figure 4

Depletion of Caspase-1 can reduce liver damage and inflammation caused by hepatic IRI. (A), (B) and (C) The hepatocellular function in serum samples was evaluated by detecting ALT, AST, and LDH levels $(n=3)$. (D) Representative images of hematoxylin and eosin (HE) staining (scale bar, $100 \mu \mathrm{m}$ ). (E) and (F) IL-1 $\beta$ and 
IL-18 were measured with ELISA in liver samples of WT and Caspase ${ }^{-1-}$ mice with Hepatic IRI and sham intervention $(n=6)$. (G) Examination of the proteolytic cleavage of Caspase-1 and GSDMD in mice liver specimens with Hepatic IRI or sham, using immunoblotting analysis. GSDMD-FL, full-length GSDMD; GSDMD-N, the N-terminal cleavage product of $\operatorname{GSDMD}(n=6)$. All data are shown as the mean $\pm \mathrm{SD}$. $* \star \star \star P<$ $0.0001 \rrbracket \star \star \star * P<0.001$.

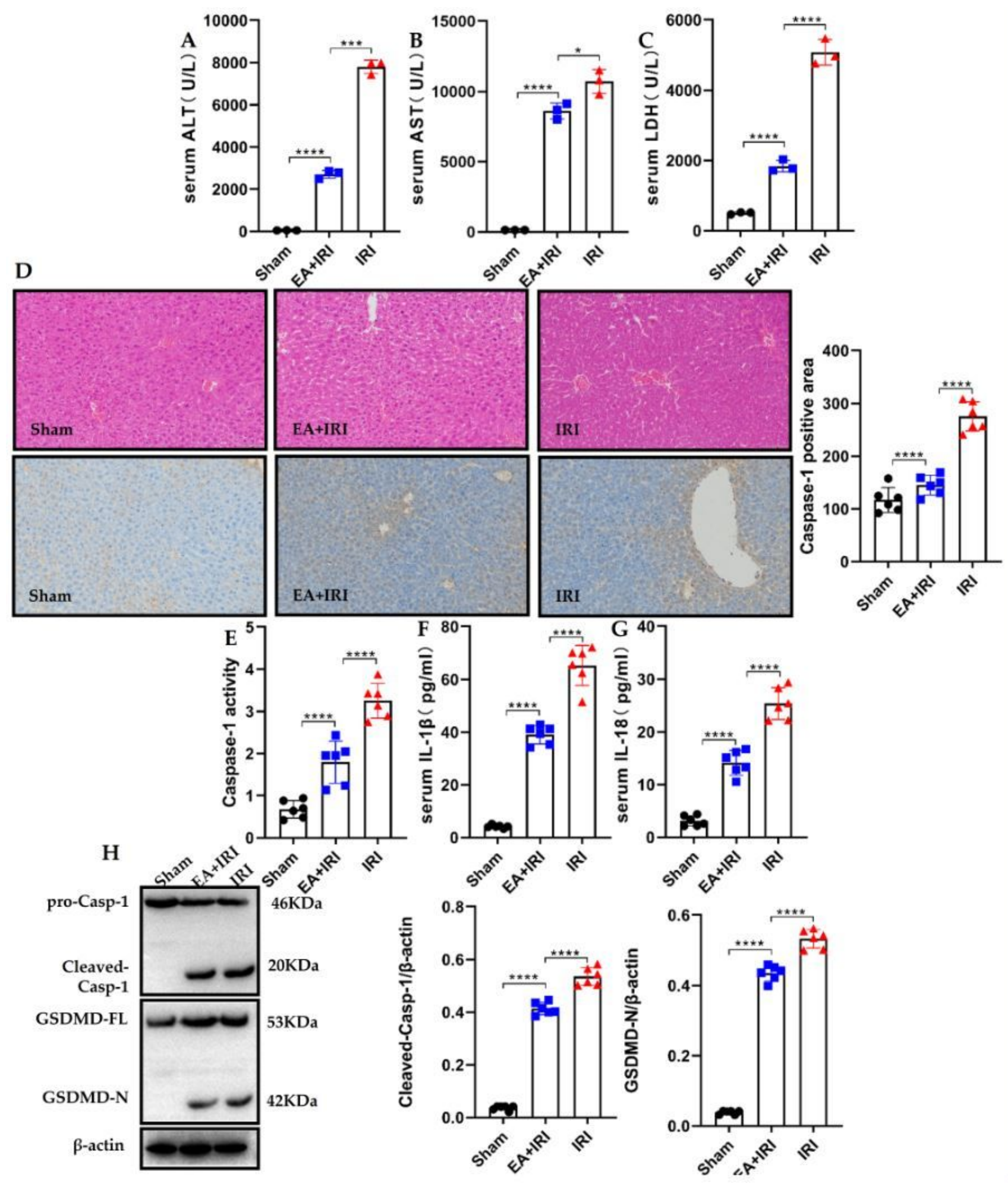

Figure 5 
Ellagic acid reduces liver injury in hepatic ischemia-reperfusion mice by inhibiting pyroptosis. (A), (B) and (C) The hepatocellular function in serum samples was evaluated by detecting ALT, AST, and LDH levels ( $n=$ 3). (D) Representative images of hematoxylin and eosin (HE) staining, and representative images of caspase-1 staining, the area of Caspase-1-positive cells in the livers were also determined (scale bar, $100 \mu m)(n=6)$. (E) and (F) IL-1 $\beta$ and IL-18 were measured with ELISA in liver samples with Hepatic IRI, sham intervention and EA treatment mice $(n=6)$. (G) Caspase-1 activities were measured in liver tissues with Hepatic IRI, sham intervention and EA treatment mice $(n=6)$. $(H)$ Examination of the proteolytic cleavage of Caspase-1 and GSDMD in mice liver specimens with hepatic IRI or sham intervention, using immunoblotting analysis. GSDMD-FL, full-length GSDMD; GSDMD-N, the N-terminal cleavage product of $\operatorname{GSDMD}(\mathrm{n}=6)$. All data are shown as the mean \pm SD. ${ }^{\star \star \star \star} P<0.0001{ }^{\star \star \star \star} P<0.001, * \star P<0.01$ and ${ }^{\star} P<0.05$.

\section{Figure 6}

A schematic diagram illustrating that pyroptosis is involved in liver ischemia-reperfusion injury, and preventive supplementation of ellagic acid can regulate pyroptosis in hepatic ischemia-reperfusion injury.

\section{Supplementary Files}

This is a list of supplementary files associated with this preprint. Click to download.

- SupplementaryMaterials.pdf 